\title{
MnSb inclusions in the GaSb matrix studied by X-ray absorption spectroscopy
}

A. Wolska ${ }^{\text {a, }}$, M.T. Klepka ${ }^{\text {a }}$, K. Lawniczak-Jablonska ${ }^{\text {a }}$, J. Sadowski ${ }^{\text {a,b }}$, A. Reszka ${ }^{\text {a }}$, B.J. Kowalski ${ }^{\text {a }}$

${ }^{a}$ Institute of Physics PAS, al. Lotnikow 32/46, PL-02-668 Warsaw, Poland

${ }^{b}$ Lund University, MAX-Lab, Lund SE-221 00, Sweden

*corresponding author:

wolska@ifpan.edu.pl

tel: (+48) 228436601 ext. 3348

fax: (+48) 228436034

\begin{abstract}
The MBE processes were performed in order to form the MnSb inclusions. Two kinds of substrates $(\mathrm{GaSb}$ and $\mathrm{GaAs})$ as well as growth temperatures $\left(450^{\circ} \mathrm{C}\right.$ and $\left.520^{\circ} \mathrm{C}\right)$ were examined. The SEM imaging was performed to determine the shape and size of formed inclusions. In all samples the inclusions of few hundred $\mathrm{nm}$ were formed. Their shapes depended strongly on the type of substrate while the size slightly increases with the growth temperature. The X-ray absorption techniques were used to monitor the local structure around $\mathrm{Mn}$ atoms and confirmed that the neighborhood of Mn atoms in the layers was close to that of the $\mathrm{MnSb}$ reference powder. We succeed in the formation of the GaSb layers with $\mathrm{Mn}$ assembled only into $\mathrm{MnSb}$ inclusions on both types of substrates: GaAs(111)A and $\mathrm{GaSb}(100)$. However, in case of the GaAs(111)A substrates, the atomic order around $\mathrm{Mn}$ atoms in $\mathrm{MnSb}$ inclusions is less perfect than in case of the $\mathrm{GaSb}(100)$ substrates.
\end{abstract}

Keywords: MnSb inclusions, MBE growth, EXAFS, SEM, room temperature ferromagnetism 


\section{Introduction}

For many years, $\mathrm{Ga}_{1-\mathrm{x}} \mathrm{Mn}_{\mathrm{x}} \mathrm{As}$ was commonly considered as a promising material for microelectronic applications utilizing the electron spin. However, studies of the uniform ternary alloy didn't succeed in significant increasing its Curie temperature $\left(\mathrm{T}_{\mathrm{C}}\right)$. The highest $\mathrm{T}_{\mathrm{C}}$ reported so far for this compound is about $190 \mathrm{~K}$ (Chen et al., 2009) which is too low for application purposes. These obstacles focused the attention on the ferromagnetic MnAs precipitates which can be quite easily produced by high temperature annealing of the $\mathrm{Ga}_{1 \text { - }}$ ${ }_{\mathrm{x}} \mathrm{Mn}_{\mathrm{x}} \mathrm{As}$ layers (Yokoyama et al., 2005; Kwiatkowski et al., 2007; Demchenko et al., 2007) or by Mn implantation (Couto et al., 2005). Such heterostructures created by introducing ferromagnetic inclusions in a semiconductor matrix became promising. In this kind of material, small ferromagnetic nanoparticles are immersed in the semiconductor host lattice providing a built-in local magnetic field. However, in case of MnAs precipitates, two crystalline structures with different magnetic properties were found: the cubic zinc blende type and hexagonal one. It means that the GaAs:(Mn,Ga)As material exhibits a ferromagnetic to superparamagnetic behavior at room temperature, depending on the inclusions' cluster size and crystallographic structure (Lawniczak-Jablonska et al., 2011a; Lawniczak-Jablonska et al., 2011c).

To overcome these obstacles, other types of precipitates, like $\mathrm{MnSb}$, are considered. Its main advantage is a $T_{C}$ well above room temperature. It was shown that bulk MnSb has a $\mathrm{T}_{\mathrm{C}}$ of $587 \mathrm{~K}$ (Panchula et al., 2003) while for MnAs it is only $318 \mathrm{~K}$. What's more, the $\mathrm{Mn}_{1-\mathrm{x}} \mathrm{Sb}_{\mathrm{x}}$ layers grown on GaAs substrate can reach a $\mathrm{T}_{\mathrm{C}}$ of $620 \mathrm{~K}$ (Akinaga et al., 1995). In contrast to MnAs, forming the MnSb inclusions by an implantation process encounters many difficulties (Wolska et al., 2010). On the other hand, studies on the MBE grown thin layers showed that already in pure material, MnSb does not form smooth surface but tends to form big islands or columns (Low et al., 1999; Lawniczak-Jablonska et al., 2009). This leads to the other advantage which is possibility to form the hexagonal MnSb precipitates directly during the MBE process without subsequent annealing. It was shown that on the $\operatorname{GaAs}(100)$ substrates the dots can be produced in the low-temperature MBE process (Mizuguchi et al., 2000; Abe et al., 2000) as well as in high temperature (Abe et al., 2000). However, based on the magnetic measurements, Abe et al., 2000 concluded that in case of low-temperature grown samples, part of $\mathrm{Mn}$ atoms is incorporated into the $\mathrm{GaSb}$ matrix forming zincblende ( $\mathrm{GaMn}) \mathrm{Sb}$ alloy.

The aim of the presented studies is to show that the properly elaborated MBE process allows to gather all $\mathrm{Mn}$ atoms into $\mathrm{MnSb}$ hexagonal inclusions and prevents the $\mathrm{Mn}$ atoms from being incorporated into GaSb or GaAs matrix. Moreover, the formation of the inclusions does not prevent the two-dimensional layer by layer growth in contrast to the GaMnAs system where MnAs segregation leads to the formation of the nanowires (Sadowski et al., 2007).

The results of microscopic and spectroscopic studies of the MnSb inclusions grown at GaAs and $\mathrm{GaSb}$ substrates at the temperatures of $450^{\circ} \mathrm{C}$ and $520^{\circ} \mathrm{C}$ are presented. The local structure around $\mathrm{Mn}$ atoms was monitored by using X-ray Absorption Near Edge Structure (XANES) and Extended X-ray Absorption Fine Structure (EXAFS) techniques. Both are the excellent tools to determine local structure around Mn atoms. Comparison of the XANES 
spectra with the standard one gives the instantaneous answer whether the desired compound was formed or not. EXAFS provides more detailed and quantitative information about the local structure. The scanning electron microscopy (SEM) was used to determine the shape and size of inclusions. Study of magnetic properties revealed that the inclusions are ferromagnetic from helium up to room temperature. It was also found out that, depending on the orientation of the substrate, the easy magnetization axis of the GaSb:MnSb granular layer was close to the in plane or out of plane directions which opens the interesting opportunities for applications in spintronic devices. Details of magnetic measurements are presented elsewhere (Lawniczak-Jablonska et al., 2011b).

\section{Experimental}

The samples were grown on the GaSb(100) and GaAs(111)A substrates using the MBE technology. Several attempts were needed in order to properly adjust the parameters of the MBE process. In all obtained samples the XANES and EXAFS spectra were used to monitor the Mn location. In many cases the MnGa inclusions or diffusion of Mn to the substrate was observed. Finally the successful MBE process was established where the MnSb inclusions were formed directly during the epitaxial growth procedure without the post growth annealing. In order to check how the type of substrate influences the dots' formation, the samples on both substrates were grown in the same process. First, the GaSb buffer of 40-45 nm was grown and then the GaMnSb layer. One set of samples was grown at $450{ }^{\circ} \mathrm{C}$ with the nominal $\mathrm{Mn}$ content of $3 \%, 8 \%$ and $11 \%$. The samples are marked respectively, as A-3/450, A-8/450 and A-11/450 for the GaAs(111)A substrate and B-3/450, B-8/450 and B-11/450 for the $\mathrm{GaSb}(100)$ one. Another set of samples was grown at $520{ }^{\circ} \mathrm{C}$ with the nominal $\mathrm{Mn}$ content of $0.5 \%, 1 \%$ and $2 \%$ and is marked as: A- $0.5 / 520, \mathrm{~A}-1 / 520$ and A-2/520 for GaAs(111)A substrate and B-0.5/520, B-1/520 and B-2/520 for the GaSb(100) one.

The XANES and EXAFS spectra at the Mn K-edge were measured at Hasylab (A1 station) at liquid nitrogen temperature using a silicon drift detector. Additional spectra of powdered MnSb standard were measured in a transmission mode. The Athena and Artemis programs (Ravel and Newville, 2005), using IFEFFIT data analysis package, were applied to the analysis of the EXAFS data. The SEM images were gathered by a scanning electron microscope Hitachi SU-70.

\section{Results and discussion}

Fig. 1 presents the SEM images of the samples grown on the $\mathrm{GaSb}(100)$ and GaAs(111)A substrates at both temperatures. As an example, samples with 3\% (Fig. 1a and c) and 2\% (Fig. 1b and d) of Mn are shown. The shapes of inclusions depend mainly on the type of the substrate. On the GaAs they are long and thin while on the GaSb they are shorter and thicker, rectangular or even square. The percentage of Mn strongly influences dots' density but not their size. However, the inclusions formed at $450{ }^{\circ} \mathrm{C}$ are smaller than those grown at $520{ }^{\circ} \mathrm{C}$. In case of the GaSb substrate the average dot's size is around $400 \times 500 \mathrm{~nm}^{2}$ for $520{ }^{\circ} \mathrm{C}$ and $300 \times 200 \mathrm{~nm}^{2}$ for $450{ }^{\circ} \mathrm{C}$. This tendency is even better visible for the GaAs 
substrate where the average size of inclusions is around $900 \times 200 \mathrm{~nm}^{2}$ for $520{ }^{\circ} \mathrm{C}$ and $500 \mathrm{x}$ $80 \mathrm{~nm}^{2}$ for $450{ }^{\circ} \mathrm{C}$.

XANES is sensitive to the local structure around a selected element; therefore, it can be treated as a fingerprint of the formed chemical bonds. The XANES spectra are directly related to the unoccupied electron density of states, which depends on the crystal symmetry. As a result, these spectra can be used to monitor the quality of the formed crystal structure. Spectra of all considered samples look very similar. In Fig. 2, as an example, the spectra for the samples presented in Fig. 1 are shown (A-3/450, B-3/450, A-2/520 and B-2/520). The MnSb standard spectrum is shown for comparison. Since all of them look close to the standard, it can be concluded that MnSb compound is formed. The small differences in the shape of the feature labeled as A can be related to the imperfection of the structure; however, the agreement between the location of the features B, C and D indicates that the lattice constant and symmetry are the same as in the standard. In order to confirm these conclusions, the EXAFS analysis was performed.

Only the first coordination shell was considered due to the fact that the higher shells are composed of many atoms located very close to each other and cannot be separated. According to the crystallographic model ( $\mathrm{P}_{3} / \mathrm{mmc}$ space group, Wyckoff, 1963), the first shell consists of 6 antimony and 2 manganese atoms at the distances of 2.78 and $2.87 \AA$, respectively. For the comparison, the standard MnSb powder spectrum was also analyzed. This enabled to determine the value of the amplitude reduction factor $\left(\mathrm{S}_{0}{ }^{2}=0.60(2)\right)$ which was then used in the fits of the MBE samples. The fit parameters are gathered in Table 1. In all cases the distances are slightly shorter than in the model (which was based on diffraction data) but equal, within an error, to those found for the standard.

The fitting was performed in the range $\sim 1.65$ to $3 \AA$. The quality of the fit is indicated by the R-factor. Usually, the value of 0.05 is taken as a limit between bad and good fit. For all of the presented fits, the R-factor is lower than 0.05 (in most cases it is one order of magnitude lower) which means that the fits are good.

The EXAFS Debye-Waller factor $\left(\sigma^{2}\right)$ consists of two terms describing the attenuation of the EXAFS signal due to the thermal and structural disorder. The part related to the thermal disorder can be minimized by performing measurements in low temperatures, as it was in this case. Therefore, it can be assumed that only the structural part, responsible for atomic displacements, plays significant role in the analysis. Fitting parameters show that for the $\mathrm{Sb}$ atoms $\sigma^{2}$ remains within an error at the same level as for the standard, except for the A$0.5 / 520$ sample where it is slightly higher. On the other hand, the R factor is almost two times larger here than for the other samples. This can be related to the low content of Mn what results in the lower quality of both the gathered data and formed crystal structure.

In case of the EXAFS Debye-Waller factor for the Mn atoms, its values are found to be considerably higher in the samples grown on the GaAs(111)A substrate than in the $\mathrm{MnSb}$ powder or even samples grown at the $\operatorname{GaSb}(100)$ substrate. Since $\sigma^{2}$ is correlated to the coordination number, it can suggest the existence of Mn vacancies or/and the increase of the ratio of the $\mathrm{Mn}$ atoms at the surface to those inside the inclusions due to a different shape of inclusions which are long and narrow. It indicates that in the inclusions formed on the $\mathrm{GaSb}(100)$ substrate the atomic order around $\mathrm{Mn}$ atoms is closer to that in bulk $\mathrm{MnSb}$ 
structure than in case of those formed on the GaAs(111)A substrate. The fitted spectra are presented in Figs. 3 and 4. The MnSb powder spectrum is shown in Fig. 6.

To demonstrate the sensitivity of the X-ray absorption method, as a comparison, the result for the sample grown in the unsuccessful processes is shown. The Mn-Ga sample was grown on the $\mathrm{GaSb}(100)$ substrate at $450{ }^{\circ} \mathrm{C}$. Fig. 5 presents XANES spectrum of the sample compared with the standard MnSb. It is clear that the shape of Mn-Ga spectrum is completely different from the standard what means that $\mathrm{MnSb}$ was not formed here.

EXAFS analysis provides more quantitative information on the sample. In Fig. 6 Fourier Transforms of the spectrum is presented. Again, the dissimilarities between Mn-Ga and $\mathrm{MnSb}$ are substantial. They differ not only in the shapes of the first shells but also in their distances to the central atom. Fitting revealed that for the Mn-Ga sample, first shell consists of the $\mathrm{Ga}$ atoms. In order to find out their number, the value of the $\mathrm{S}_{0}{ }^{2}$ parameter had to be estimated. Since only one type of atoms is present here, it is possible to use method described in Ravel, 2000. This resulted in the $\mathrm{S}_{0}{ }^{2}$ equal to 0.91 and the number of $\mathrm{Ga}$ atoms equal to 6.4(1.3) within the distance of 2.59(1) A. High number of the Ga neighbors and lack of the second shell mean that $\mathrm{Mn}$ atoms are not built in the GaSb matrix but they are randomly distributed in the Ga-based inclusions. Apparently, in this case the Sb flow was not high enough and the MnSb compound couldn't be formed.

\section{Conclusions}

In summary, four series of samples prepared on the $\mathrm{GaSb}(100)$ and $\mathrm{GaAs}(111) \mathrm{A}$ substrates at $450{ }^{\circ} \mathrm{C}$ and $520{ }^{\circ} \mathrm{C}$ were presented here. In all samples the inclusions of few hundred nm were formed, as shown by SEM imaging. Their shapes depended strongly on the type of substrate while the size slightly increased with the growth temperature. The Mn concentration influences mainly the density of formed inclusions. The X-ray absorption techniques helped in determining the atomic local structure around $\mathrm{Mn}$ atoms, showing that in all cases the MnSb compound was formed. However, in case of the GaAs(111)A substrates, the atomic order around $\mathrm{Mn}$ atoms in $\mathrm{MnSb}$ inclusions is less perfect than in case of the $\mathrm{GaSb}(100)$ substrates.

\section{Acknowledgments}

This work was partially supported by national grant of Ministry of Science and High Education N202-052-32/1189. The research leading to these results has received funding from the European Community's Seventh Framework Programme (FP7/2007-2013) under Grant agreement no. 226716.

\section{References}

Abe, E., Matsukura, F., Yasuda, H., Ohno, Y., Ohno, H., 2000. Molecular beam epitaxy of III-V diluted magnetic semiconductor (Ga,Mn)Sb. Physica E, 7, 981-985. 
Akinaga, H., Tanaka, K., Ando, K., Katayama, T., 1995. Fabrication and magneto-optical properties of epitaxial ferromagnetic $\mathrm{Mn}_{1-\mathrm{x}} \mathrm{Sb}$ thin films grown on GaAs and sapphire. J. Cryst. Growth 150, 1144-1449.

Chen, L., Yan, S., Xu, P.F., Lu, J., Wang, W.Z., Deng, J.J., Qian, X., Ji, Y., Zhao, J.H., 2009. Low-temperature magnetotransport behaviors of heavily Mn-doped (Ga,Mn)As films with high ferromagnetic transition temperature. Appl. Phys. Lett. 95, 182505 (3pp).

Couto, O.D.D., Jr., Brasil, M.J.S.P., Iikawa, F., Giles, C., Adriano, C., Bortoleto, J.R.R., Pudenzi, M.A.A., Gutierrez, H.R., Danilov, I., 2005. Ferromagnetic nanoclusters formed by Mn implantation in GaAs. Appl. Phys. Lett. 86, 071906 (3pp).

Demchenko, I.N., Lawniczak- Jablonska, K., Story, T., Osinny, V., Jakiela, R., Domagala, J.Z., Sadowski, J., Klepka, M., Wolska, A,. Chernyshova, M., 2007. Modification of the local atomic structure around $\mathrm{Mn}$ atoms in $(\mathrm{Ga}, \mathrm{Mn})$ As layers by high temperature annealing. J. Phys.: Condens. Matt. 19, 496205 (14pp).

Kwiatkowski, A., Wasik, D., Kamńska, M., Bożek, R., Szczytko, J., Twardowski, A., Borysiuk, J., Sadowski, J., Gosk, J., 2007. Structure and magnetism of MnAs nanocrystals embedded in GaAs as a function of post-growth annealing temperature. J. Appl. Phys. 101, 113912 (6pp).

Lawniczak-Jablonska, K., Wolska, A., Bak-Misiuk, J., Dynowska, E., Romanowski, P., Domagała, J.Z., Minikayev, R., Wasik, D., Klepka, M.T., Sadowski, J., Barcz, A., Dluzewski, P., Kret, S., Twardowski, A., Kamińska, M., Persson, A., Arvanitis, D., Holub-Krappe, E., Kwiatkowski, A., 2009. Structural and magnetic properties of MBE grown MnSb layers on GaAs substrates. J. Appl. Phys. 106, 083524 (13pp).

Lawniczak-Jablonska, K., Libera, J., Wolska, A., Klepka, M.T., Dluzewski, P., Sadowski, J., Wasik, D., Twardowski, A., Kwiatkowski, A., Sato, K., 2011a. The source of room temperature ferromagnetism in granular GaMnAs layers with zinc blende clusters. Phys. Status Solidi RRL 5, 62-64.

Lawniczak-Jablonska, K., Wolska, A., Klepka, M.T., Kret, S., Gosk, J., Twardowski, A., Wasik, D., Kwiatkowski, A., Kurowska, B., Kowalski, B.J.,Sadowski, J., 2011 b. Magnetic properties of $\mathrm{MnSb}$ inclusions formed in $\mathrm{GaSb}$ matrix directly during molecular beam epitaxial growth. J. Appl. Phys. 109, 074308 (7pp).

Lawniczak-Jablonska, K., Bak-Misiuk, J., Dynowska, E., Romanowski, P., Domagala, J.Z., Libera, J., Wolska, A., Klepka, M.T., Dluzewski, P., Sadowski, J., Barcz, A., Wasik, D., Twardowski, A., Kwiatkowski, A. 2011c. Structural and magnetic properties of nanoclusters in GaMnAs granular layers. J. Solid State Chem., accepted for publication.

Low, B.L., Ong, C.K., Lin, J., Huan, A.C.H., Gong, H., Liew, T.Y.F., 1999. Structure and magnetization of $\mathrm{MnSb}$ thin films deposited at different substrate temperatures. J. Appl. Phys. 85, 7340-7344.

Mizuguchi, M., Akinaga, H., Ono, K., Oshima, M., 2000. Formation and structural investigation of MnSb dots on S-passivated GaAs( $\begin{aligned} & 0 \\ & 0\end{aligned}$ 1) substrates. J. Cryst. Grow. 209, 552-555.

Panchula, A.F., Kaiser, C., Kellock, A., Parkin, S.S., 2003. Spin polarization and magnetotransport of $\mathrm{Mn}-\mathrm{Sb}$ alloys in magnetic tunnel junctions. Appl. Phys. Lett. 83, 1812-1814.

Ravel, B., 2000. EXAFS Analysis with FEFF and FEFFIT, Part 2, Commentary 37. 
Ravel, B., Newville, M., 2005. ATHENA, ARTEMIS, HEPHAESTUS: data analysis for Xray absorption spectroscopy using IFEFFIT. J. Synchrotron Rad. 12, 537-541.

Sadowski, J., Dłużewski, P., Kret, S., Janik, E., Łusakowska, E., Kanski, J., Presz, A., Terki, F., Charar, S., Tang, D., 2007. GaAs:Mn nanowires grown by molecular beam epitaxy of (Ga,Mn)As at MnAs segregation conditions. Nano Lett. 7, 2724-2728.

Wolska, A., Lawniczak-Jablonska, K., Klepka, M.T., Barcz, A., Hallen, A., Arvanitis, D., 2010. Study of the local environment of Mn ions implanted in GaSb. Acta Physica Polonica A 117, 286-292

Wyckoff, R.W.G., 1963. Crystal Structures. Second edition. Interscience Publishers, New York 1, 85-237.

Yokoyama, M., Yagamuchi, H., Ogawa, T., Tanaka, M., 2005. Zinc-blende-type MnAs nanoclusters embedded in GaAs. J. Appl.Phys. 97, 10D317 (3pp).

\section{Table 1.}

The fitting parameters of the EXAFS spectra of the MnSb standard and the samples grown on the $\mathrm{GaSb}(100)$ substrate at $520^{\circ} \mathrm{C}(B-* / 520)$ and $450^{\circ} \mathrm{C}(B-* / 450)$ as well as the samples grown on the $\mathrm{GaAs}(111) \mathrm{A}$ substrate at $520^{\circ} \mathrm{C}(A-* / 520)$ and $450^{\circ} \mathrm{C}(A-* / 450)$. $\mathrm{R}$ - a distance to the central atom, $\sigma^{2}$ - EXAFS Debye-Waller factor, R-factor indicates quality of the fit.

\begin{tabular}{|c|c|c|c|c|c|}
\hline & $\mathrm{R}_{\mathrm{Sb}}[\AA]$ & $\mathrm{R}_{\mathrm{Mn}}[\AA]$ & $\sigma_{\mathrm{Sb}}{ }^{2}\left[\AA^{2}\right]$ & $\sigma_{\mathrm{Mn}}^{2}\left[\AA^{2}\right]$ & $\mathrm{R}$-factor \\
\hline $\mathrm{MnSb}$ & $2.76(1)$ & $2.86(1)$ & $0.007(1)$ & $0.007(2)$ & 0.003 \\
\hline$B-0.5 / 520$ & $2.76(2)$ & $2.86(2)$ & $0.007(1)$ & $0.008(4)$ & 0.012 \\
\hline$B-1 / 520$ & $2.76(1)$ & $2.86(1)$ & $0.006(1)$ & $0.008(3)$ & 0.002 \\
\hline$B-2 / 520$ & $2.77(1)$ & $2.87(1)$ & $0.007(1)$ & $0.006(7)$ & 0.002 \\
\hline$B-3 / 450$ & $2.76(1)$ & $2.87(1)$ & $0.007(1)$ & $0.007(3)$ & 0.002 \\
\hline$B-8 / 450$ & $2.76(1)$ & $2.87(1)$ & $0.006(1)$ & $0.009(5)$ & 0.008 \\
\hline$B-11 / 450$ & $2.76(1)$ & $2.87(1)$ & $0.005(1)$ & $0.005(2)$ & 0.007 \\
\hline$A-0.5 / 520$ & $2.73(2)$ & $2.84(2)$ & $0.010(2)$ & $0.014(8)$ & 0.040 \\
\hline$A-1 / 520$ & $2.76(1)$ & $2.87(1)$ & $0.009(1)$ & $0.011(4)$ & 0.018 \\
\hline$A-2 / 520$ & $2.77(1)$ & $2.88(1)$ & $0.009(1)$ & $0.010(3)$ & 0.009 \\
\hline$A-3 / 450$ & $2.76(2)$ & $2.86(2)$ & $0.007(1)$ & $0.011(7)$ & 0.014 \\
\hline$A-8 / 450$ & $2.75(1)$ & $2.86(1)$ & $0.007(1)$ & $0.016(7)$ & 0.009 \\
\hline$A-11 / 450$ & $2.76(1)$ & $2.87(1)$ & $0.008(1)$ & $0.015(5)$ & 0.009 \\
\hline
\end{tabular}




\section{Figure Captions:}

\section{Fig. 1.}

SEM images of the samples with $3 \%$ (grown at $450^{\circ} \mathrm{C}$ ) and $2 \%$ (grown at $520^{\circ} \mathrm{C}$ ) of $\mathrm{Mn}$ : a) $A$ $3 / 450$; b) $A-2 / 520$; c) $B-3 / 450$;

\section{Fig. 2.}

Normalized XANES spectra of the selected samples with $2 \%$ and $3 \%$ of Mn compared with the MnSb standard. Spectra are shifted vertically for clarity.

\section{Fig. 3.}

Results of the fitting (in R space) of the EXAFS spectra of the samples grown on the $\mathrm{GaSb}(100)$ substrate. Spectra are shifted vertically for clarity. Dotted lines represent experiment, full lines - obtained fits.

\section{Fig. 4.}

Results of the fitting (in R space) of the EXAFS spectra of the samples grown on the GaAs(111)A substrate. Spectra are shifted vertically for clarity. Dotted lines represent experiment, full lines - obtained fits.

\section{Fig. 5.}

Normalized XANES spectra of the MnSb standard compared with the Mn-Ga sample.

\section{Fig. 6.}

Fitting results of the MnSb standard and the $M n-G a$ sample where the first shell is formed by $\mathrm{Ga}$ atoms only. Spectra are shifted vertically for clarity. Dotted lines represent experiment, full lines - obtained fits 


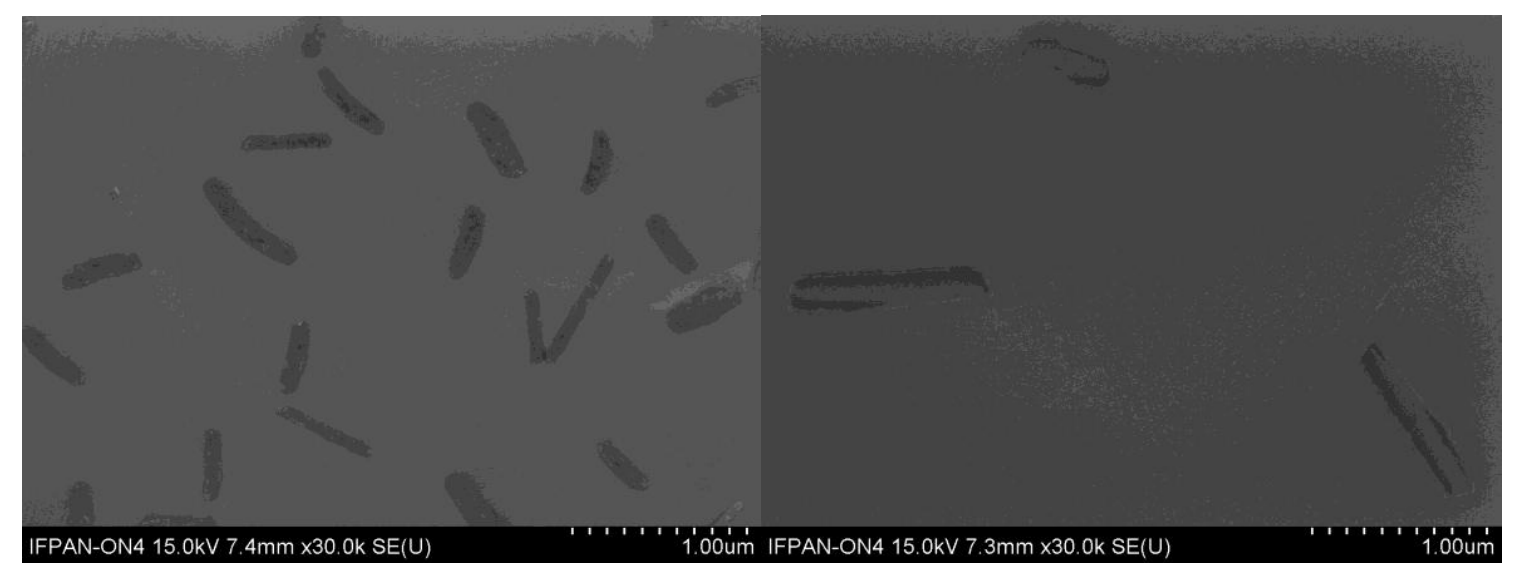

a) b)

c)

\section{d)}

Figure 1.

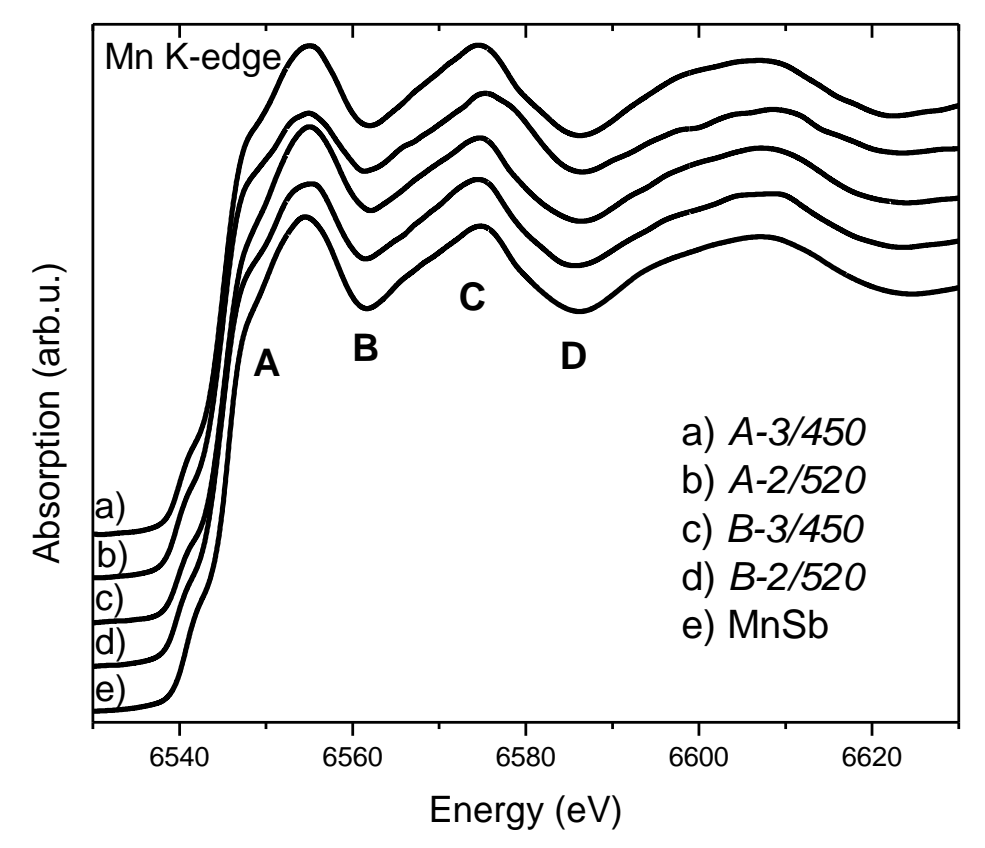

Figure 2. 


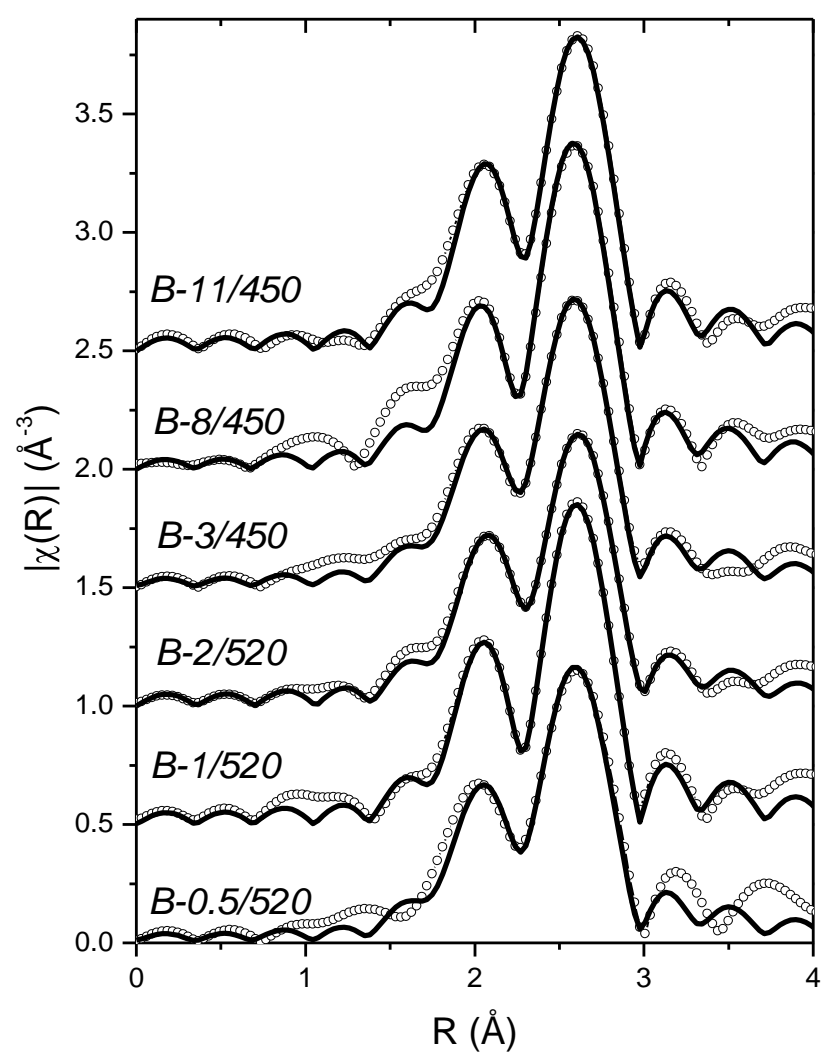

Figure 3. 


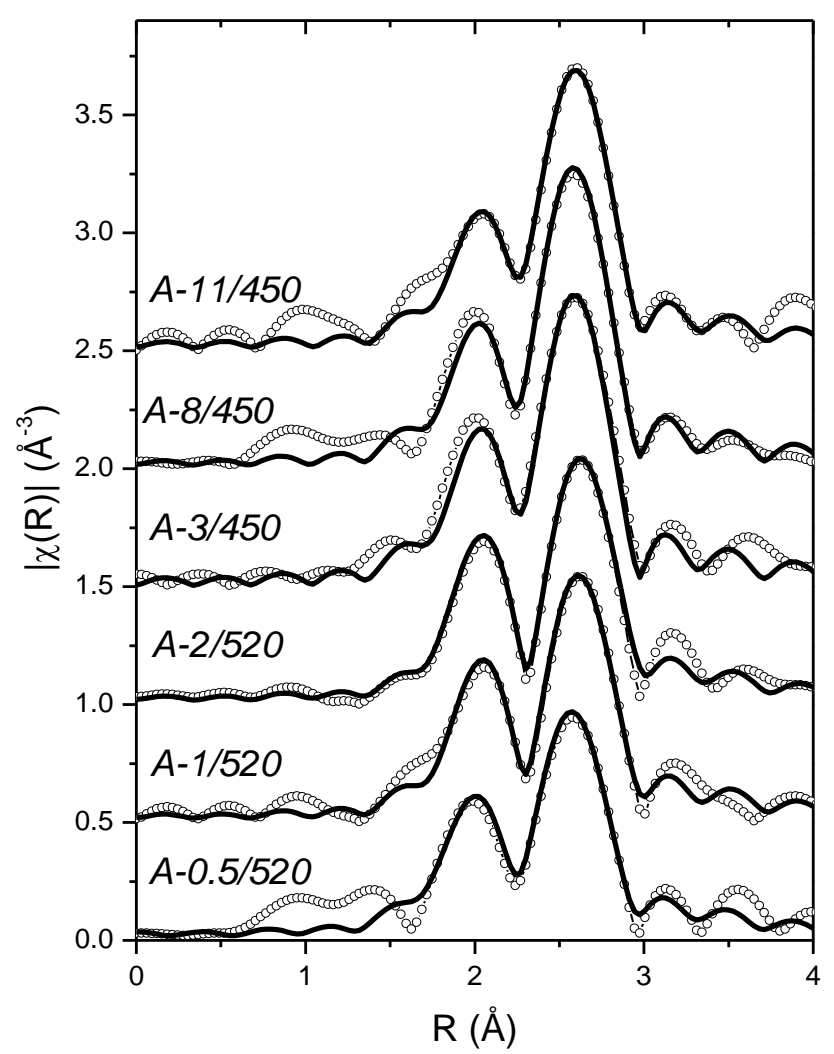

Figure 4.

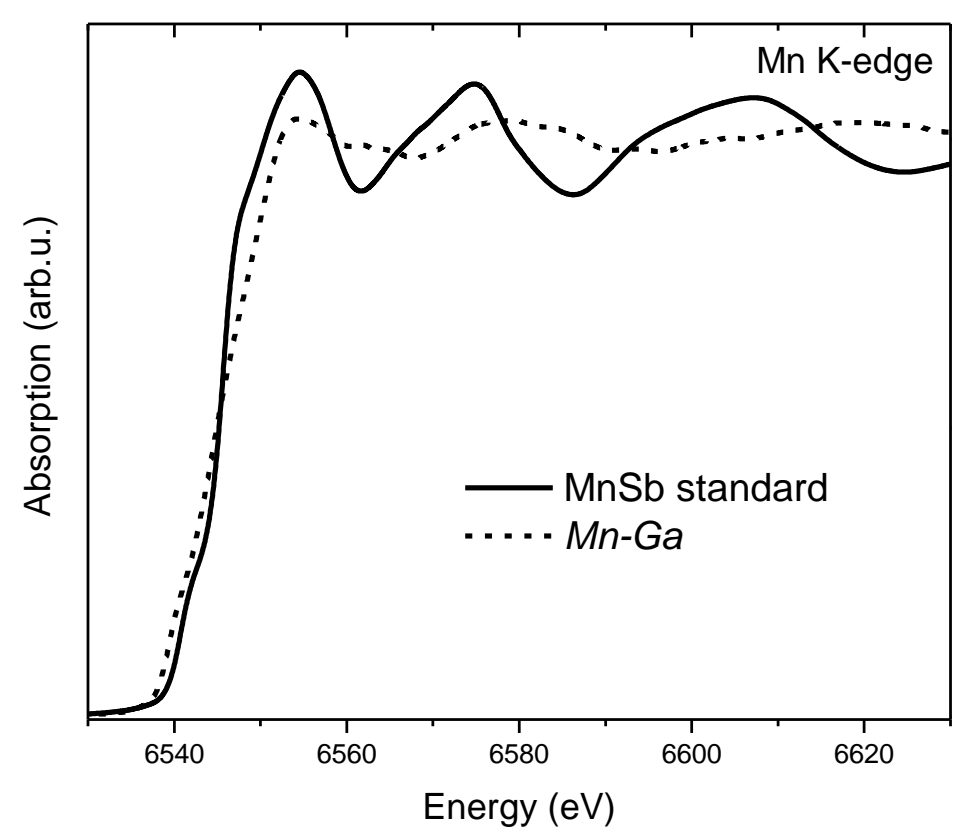

Figure 5. 


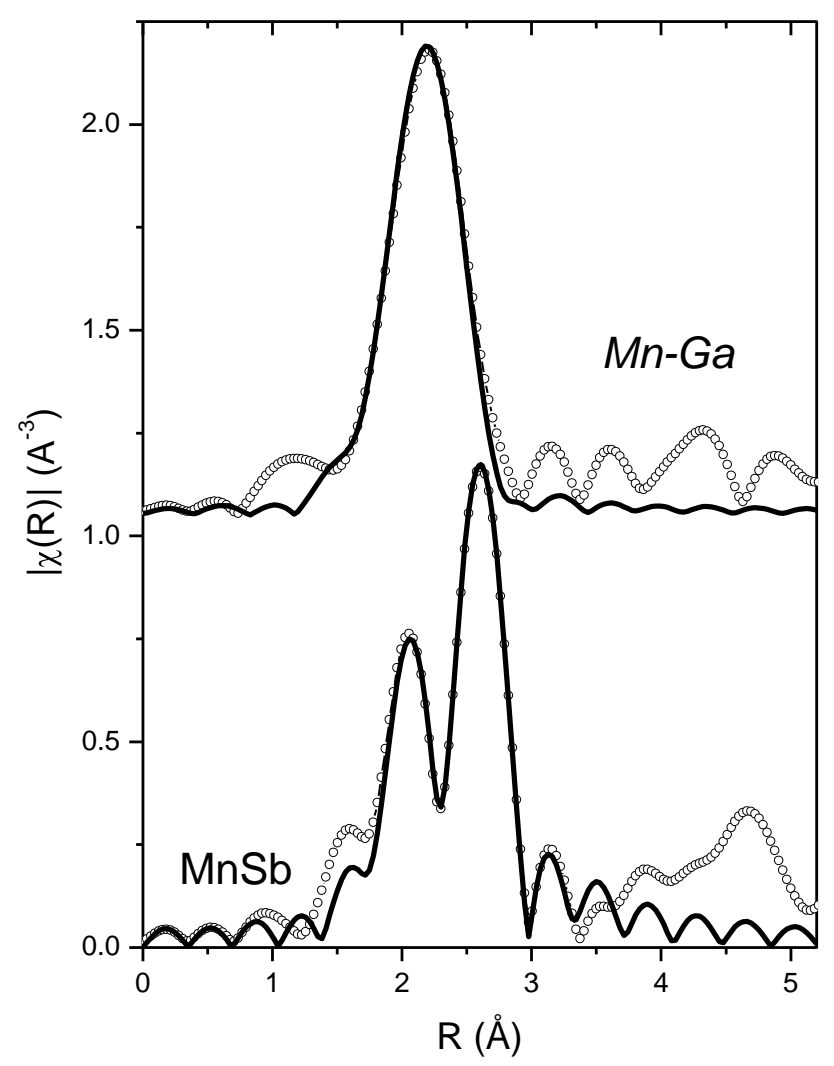

Figure 6. 